\title{
Seeing multiple directions of motion-physiology and psychophysics
}

\author{
Stefan Treue, Karel Hol and Hans-Jürgen Rauber
}

Cognitive Neuroscience Laboratory, Dept. of Neurology, University of Tübingen, Auf der Morgenstelle 15, 72076 Tübingen, Germany

Correspondence should be addressed to S.T. (treue@uni-tuebingen.de)

\begin{abstract}
Dot patterns sliding transparently across one another are normally perceived as independently moving surfaces. Recordings from direction-selective neurons in area MT of the macaque suggested that this perceptual segregation did not depend on the presence of two peaks in the population activity. Rather, the visual system seemed to use overall shape of the population response to determine the number and directions of motion components. This approach explained a number of perceptual phenomena, including susceptibility of the motion system to direction metamers, motion patterns combining three or five directions incorrectly perceived by subjects as comprising only two directions. Our findings offer insights into the coding of multi-valued sensory signals and provide constraints for biologically based computational models.
\end{abstract}

Analysis of visual motion is an important aspect of processing visual information. It is therefore not surprising that primates have cells and cortical areas specialized for visual motion processing. The specialized neurons are direction selective: each responds only to a particular subset of directions and speeds of motion within its receptive field. Cortical areas along the dorsal pathway of the primate visual system are specialized for the analysis of visual motion; most notably, these include area MT, which is dominated by direction-selective cells, and area MST, which also contains cells encoding more complex motions like rotation or expansion ${ }^{1}$. MT and MST are arguably the most intensively studied extrastriate cortical areas. The curve facing out and left in Fig. 1a is an idealized tuning curve of an MT cell to a random dot pattern moving in a single direction. The response distribution is bell shaped and is well fit by a Gaussian curve. As is characteristic of direction-selective neurons throughout the visual system, responses are broadly tuned, with a tuning width of approximately $90^{\circ}$, on average ${ }^{2-4}$. Although neurons differ in their preferred directions, their tuning curves are otherwise very similar. If typical tuning curves are combined and plotted as a function of direction preference, we obtain a three-dimensional graph of population responses as a function of both stimulus direction and preferred directions of the neurons (Fig. 1a). Experimentally, this threedimensional plot of activity can be derived by determining responses of individual cells to movement in different directions (contour lines in Fig. 1a). The brain, however, must calculate this function from information derived from the opposite scenario: a single stimulus simultaneously activates a population of neurons, each preferring a different direction.

We can predict this population response to a single stimulus from the three-dimensional plot by taking neural responses along a line parallel to the axis denoting direction preference (the curve facing right in Fig. 1a). This population response has the same shape as the tuning curve for an individual cell. We will there- fore use the terms tuning curve (which describes a single neuron's response to many directions of motion) and population response (which describes the response of a population of neurons tuned to different directions of motion to one stimulus) interchangeably.

The bell-shaped distribution of population activity suggests that the visual system might recover the direction of a stimulus by determining the most active neurons. Such an approach of taking the preferred direction of the most active of a population of direction-tuned neurons as the perceived direction may explain the ability of the visual system to extract the overall direction of motion even for displays combining more than one direction ${ }^{5,6}$. Even for these displays, activity is greatest for neurons whose preferred directions match the center of the distribution of stimulus directions.

Not all visual motion displays create a perception of unidirectional motion, though. Combination of two patterns sliding past each other often creates the impression of two separate surfaces, each moving in a distinct direction. If the percept of a distinct direction is based on the existence of a peak in the population activity, then the perception of transparent motion implies the presence of two peaks. This is either assumed in or a feature of several models of motion perception ${ }^{7-9}$ and cortical motion processing ${ }^{10,11}$.

If we hypothesize that the response to a transparent stimulus is simply the scaled sum of the activities evoked by the individual components ${ }^{12,13}$, we can generate a hypothetical curve for population activity in response to movement in two directions at a large angle (Fig. 1c). (Several studies suggest that the response to two motions is the average of the individual responses, giving a scaling factor of 0.5. Note, however, that the exact value is not critical, as multiplication by any factor gives the same shape for the population activity as the unscaled sum of the two individual responses.) However, if the two directions of motion form such a small angle that the two peaks merge (Fig. 1d), the percept 


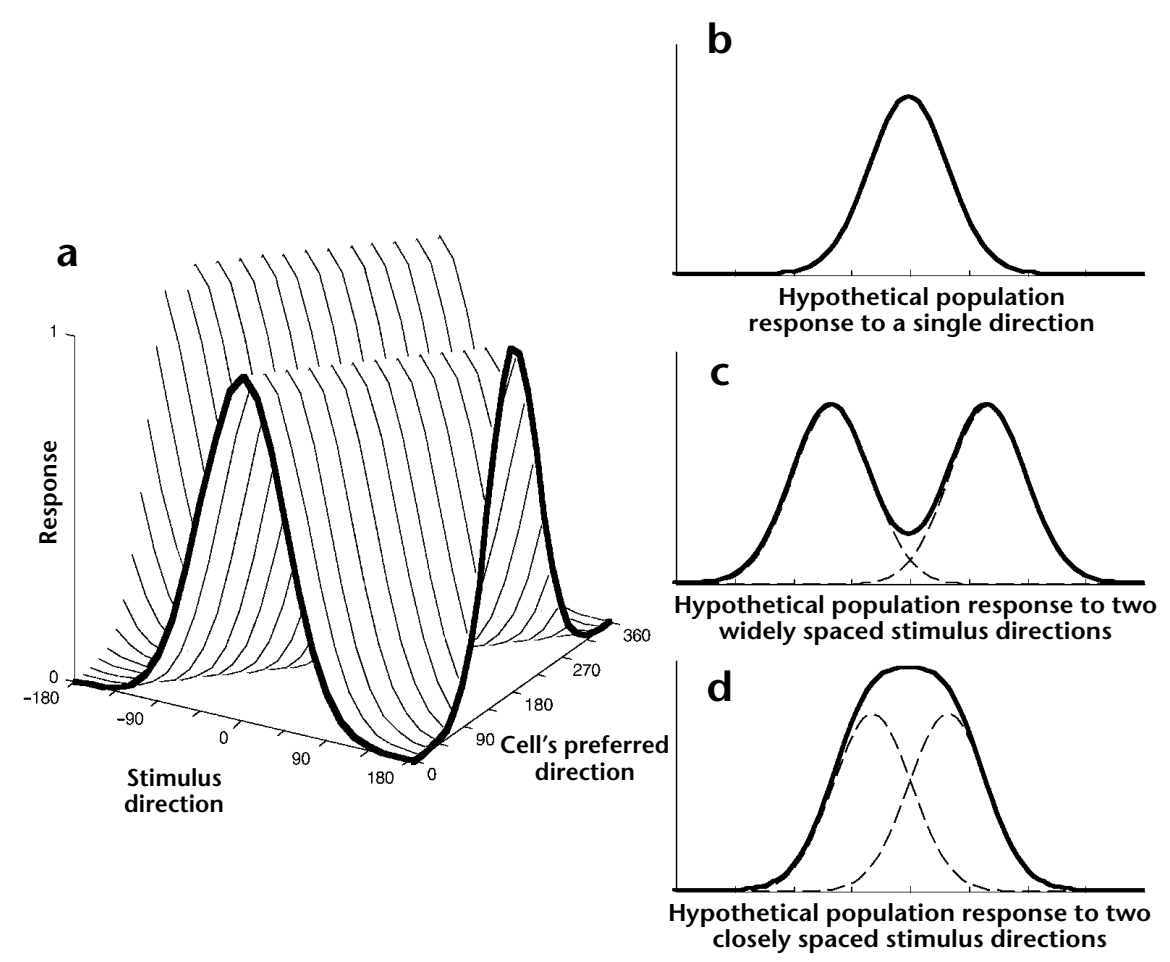

Fig. I. Direction-tuning curves and hypothetical population responses to transparent patterns. (a) A series of hypothetical tuning curves plotted three-dimensionally as a function of stimulus direction ( $x$ axis) and the preferred direction of a cell ( $y$ axis). The $z$ axis plots responses normalized to the maximal response (response to the preferred direction). The curve facing out and left is the response curve of a neuron tuned to $0^{\circ}$, and the curve facing to the right shows the population response to a stimulus moving at $180^{\circ}$. (b) A hypothetical population response to a single direction of motion. (c) A hypothetical response to movement of two transparent stimuli at an angle large enough to produce a double-peaked population response. The dashed lines indicate the population responses to each of the two directions. (d) Response as in (c) but with an angle small enough to merge the two curves into a single-peaked population response.

should change from transparent motion to motion in a single direction intermediate to the two actual component directions.

Perceptually, the transition from perceiving two separate directions to only one direction occurs when the two superimposed transparent random dot patterns move at a relative angle of about $10^{\circ}$ or less ${ }^{14}$. Although several physiological studies examine responses of direction-selective neurons to transparent patterns, little is known about the population activities evoked by patterns combining similar directions, as almost all of these experiments involve only a small subset of directions or directions at large relative angles.

The hypothesis that the profile of responses to motion in multiple directions is the scaled sum of the responses to the individual components makes a clear prediction: the sum of two Gaussians shows distinct peaks if the distance between the centers of each distribution exceeds their width (which for directiontuned neurons averages about $90^{\circ}$ ). Conversely, transparent motion at any acute angle should yield an activity profile with a single peak. This means that either the response profile to two directions is a highly non-linear combination of the individual directions, resulting in two peaks even for small directional separations between the components, or that a percept of transparent motion does not depend on the presence of two peaks in the population activity.

Here we show that the latter seems to be the case. Perception of multidirectional motion does not require distinct peaks in the population activity. Rather, the visual system seems to be able to interpret the overall shape of the activity profile. This allows the visual system to segregate motions differing even by only a small angle. However, we also show that summing the responses to the individual motion components makes the motion system susceptible to direction metamers, stimuli that are perceptually indistinguishable even though they contain different direction components.

\section{RESULTS}

We recorded the responses of 152 direction-selective cells in area MT of 3 macaques to moving random dot patterns while the monkeys performed a fixation task. The stimuli moved coherently within a stationary virtual aperture either in one direction or in two directions separated by $30^{\circ}, 60^{\circ}, 90^{\circ}$ or $120^{\circ}$; the 5 resulting activity profiles are shown for a narrowly tuned example cell (Fig. 2a). The top panel plots the responses for single motion. The line represents the best fitting Gaussian (tuning width, $58^{\circ}$ ). Given the tuning width of this neuron, the scaled sum of the responses to the individual components of the transparent motion predicts a single-peaked activity profile for the $30^{\circ}$ pattern and two peaks for the $90^{\circ}$ and $120^{\circ}$ stimuli (Fig. 2a, lower panels). As predicted, two peaks were generated only for stimuli separated by $90^{\circ}$ or $120^{\circ}$. To test our prediction for our entire sample of neurons, we aligned every activity profile to the cells' preferred direction, normalized its height to the response to that direction and then averaged all profiles (Fig. 2b). The tuning width of the averaged responses to the single-direction stimulus was $96^{\circ}$; therefore, as predicted, the activity profiles did not show two peaks for stimuli at acute angles, remaining essentially flat for the $90^{\circ}$ stimulus. Note, though, that because tuning width varied somewhat between neurons, we were concerned that deviations from our prediction might be occluded by averaging across neurons irrespective of their tuning width. Therefore, we recoded the data to test our prediction more directly. For every cell, responses were expressed as a function of the neuron's tuning width to single directions. Similarly, the angles between transparent stimuli were also expressed as fractions of the width of a cell's tuning curve to a single direction. Averaging across all cells generated a contour plot (Fig. 2c). The $x$ axis plots the average stimulus direction as a proportion of the tuning width, the $y$ axis gives the normalized angle formed by the two directions in the stimulus, and the contour surface represents the 


\section{articles}
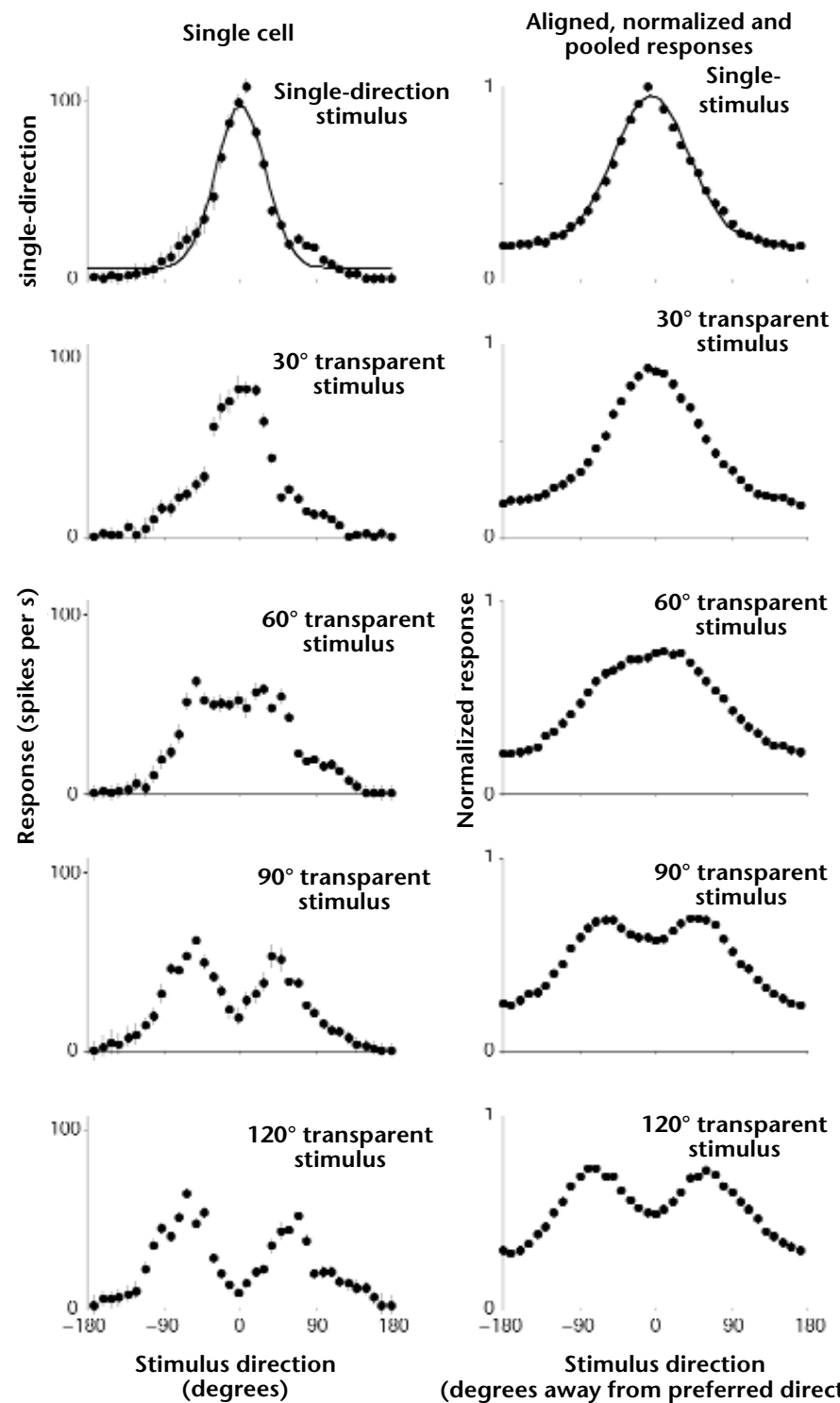

Stimulus direction
(degrees away from preferred direction)

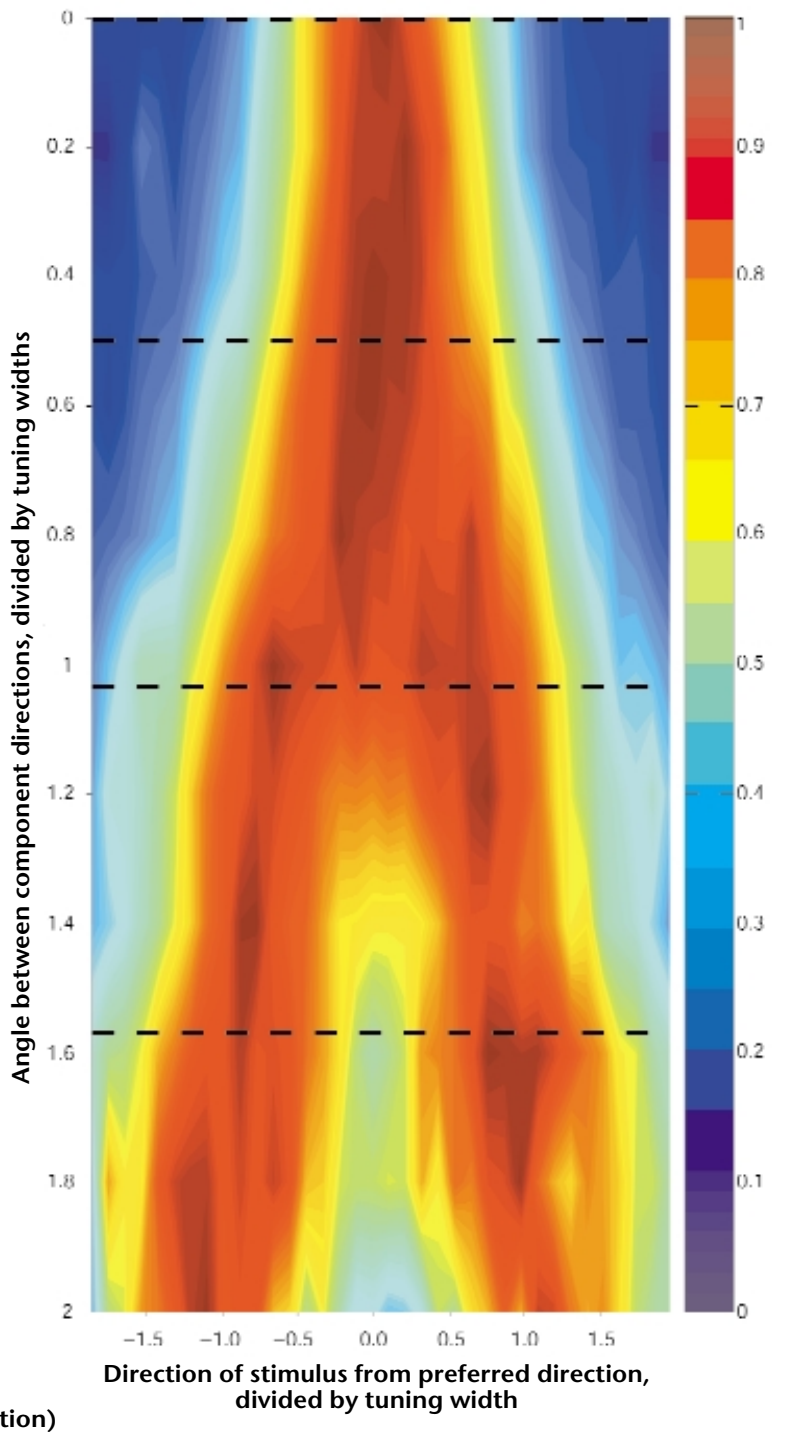

Fig. 2. Population activity profiles for transparent motion of various angles. (a) Response profiles for an example cell to the single-direction stimulus (top curve) and the bidirectional stimuli at $30^{\circ}, 60^{\circ}, 90^{\circ}$ and $120^{\circ}$ (bottom 4 curves). The $x$ axis plots the stimulus direction relative to the cell's preferred direction. For the transparent motion patterns, the stimulus direction plotted is the average of the two direction components. (b) Response profiles averaged across all cells after normalizing the firing rates to the response to the preferred single direction (determined by fitting with a Gaussian curve). (c) Contour plot of the responses across all cells. The data from each neuron are normalized to the highest response in each response profile. The resulting data points form a horizontal line in the graph at the $y$ position defined by the angle between the two directions present in the stimulus normalized by the width of the neuron's tuning curve for single directions. The $x$ axis similarly expresses the angle of the stimulus direction relative to the neuron's single-direction tuning width. The four horizontal dashed lines indicate data for the example cell from the left column. From top to bottom, they show data points for the single direction, $30^{\circ}, 60^{\circ}$ and $90^{\circ}$ stimulus conditions on the contour plot. Because of the narrow tuning of the example cell, the data from the $120^{\circ}$ stimulus condition fell below the scale. The data are in agreement with our prediction that the population activity splits into two peaks when the stimulus angle exceeds the bandwidth (when $y>1$ ).

normalized firing rate. The graph forms an inverted ' $\mathrm{Y}$ ', clearly showing that for ratios less than one (when the angle between stimuli is smaller than the neuron's tuning width), the response profile is single-peaked, whereas there are two peaks for larger angles.

This demonstrates that the simple scaled sum of the responses to individual components accounts for responses to multidirectional stimuli very well. Thus, the response profile was not multi-peaked when the angle between component directions was at the tuning width of $\sim 90^{\circ}$ or smaller. Nevertheless, the visual system can recover the directions underlying transparent motion with much smaller directional separation. How is this remarkable ability achieved? Note that, although the population activity was not multi-peaked, it nevertheless contained information about the underlying directions. Single-lobed activity profiles generated by bidirectional stimuli are notably broader than tuning curves for a single direction (Figs. 1d and 2). This change in overall shape of the activity profile might be used by the visual 


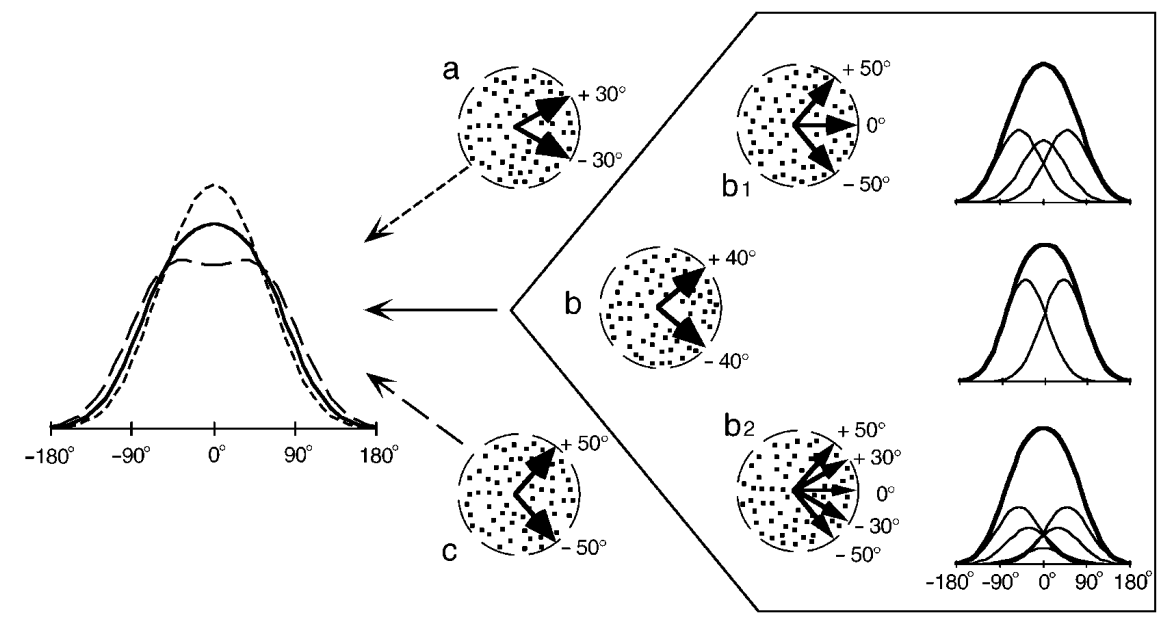

Fig. 3. Psychophysical stimuli. We created two stimuli with three $\left(\mathbf{b}_{1}\right)$ and five $\left(\mathbf{b}_{2}\right)$ directions that should be directional metamers to the $\pm 40^{\circ}$ bidirectional stimulus (b). The three and five directions and the corresponding dot densities were chosen such that the sum of the individual curves (shown along the right) was virtually identical to the activity evoked by the $\pm 40^{\circ}$ bidirectional stimulus. If the population activity was indeed the one proposed here and the only information available to the motion system, these three stimuli $\left(\mathbf{b}, \mathbf{b}_{1}, \mathbf{b}_{\mathbf{2}}\right)$ should appear to contain the same direction components. In addition to the potentially metameric bidirectional stimulus (b), we created two other bidirectional stimuli ( $\mathbf{a}$ and $\mathbf{c}$ ). These cause population activities different from the three potential metamers (as shown in the left graph) and should therefore be perceptually distinguishable from the other stimuli. Note that the directions in the $\pm 50^{\circ}$ bidirectional stimulus (c) are present as the steepest directional components in stimuli $b_{1}$ and $b_{2}$. Because the subjects were asked to judge the most upward component in all stimuli, stimuli $b_{1}$ and $b_{2}$ should result in the same responses as the $\pm 50^{\circ}$ stimulus if the motion system correctly encoded the individual motion components. Tuning width for the response profiles used here was assumed to be $90^{\circ}$, although note that the width of the summed activity for the three- and five-direction stimuli $\left(\mathbf{b}_{1}\right.$ and $\left.\mathbf{b}_{\mathbf{2}}\right)$ remains close to the activity width evoked by the two-directional stimulus (b) for a large range of tuning-curve widths. Further material and demonstrations of our stimuli can be viewed at http://neurosci.nature.com/web_specials/.

system to identify direction(s) evoking the activity. Component directions of a bidirectional stimulus can even be approximated (to within $\pm 10^{\circ}$ ) by simply subtracting a cell's tuning width for a single-direction stimulus from the width of the response profile to the given bidirectional stimulus.

Note that such an approach is different from vector averaging, which recovers a direction by calculating the vector sum of the preferred directions of all activated neurons, weighted by the respective activities of those neurons. Such an approach would yield an intermediate direction whenever two directions are combined. In contrast, a winner-take-all approach predicts perceived direction as the preferred direction of the most active neurons ${ }^{15}$. Note that, again, transparently moving patterns would be perceived as having a single direction of motion, and, as our data show, this direction would be equivalent to that predicted by the vector-averaging model if the two actual directions of motion formed an acute angle. At larger angles, the population activity shows two peaks, and the winner-take-all model would pick one of the two peaks. Clearly, this cannot account for the perception of two directions of motion when they form either a large angle or a perceptually separable acute angle (larger than $\sim 10^{\circ}$ ).

We suggest that the visual system uses another approach to recover the direction(s) underlying a given population activity across direction-selective neurons. The perceived direction(s) represent the position(s) of the smallest number of Gaussianshaped activity profiles (with widths equal to that for the aver- age direction-tuning curve) that must be summed to produce the observed activity profile. For motion in a single direction, this would be a single Gaussian positioned at the actual direction. When movement in two directions is presented, the resulting population activity is too broad to be accounted for by a single Gaussian, but can be described with two Gaussians. This approach would yield the correct percept even when the component directions form an acute angle and, therefore, result in a singlepeaked activity profile (Fig. 1d). Such an approach can be modeled formally (see Discussion).

Although this approach is normally a robust method of recovering the underlying direction(s), it should fail to distinguish between different stimuli when they evoke the same activity profile. Such metamers, stimuli that are perceptually indistinguishable despite actual differences, can be used to psychophysically estimate direction-tuning bandwidth ${ }^{16}$. If the visual system indeed recovers direction components of transparent motion by assessing the width or overall shape of the population response, it should perceive only two directions in patterns containing three or five directions, as long as the resulting profile of the population activity matches that expected for two directions of motion (Fig. 3).

In a series of psychophysical experiments, human subjects were asked to report the number of directions perceived in these patterns under eccentric fixation. In more than $94 \%$ of the trials with stimuli containing 3 directions, subjects reported perceiving two directions. Even the stimulus with 5 directions was reported to contain just 2 directions in more than $73 \%$ of the trials. We proceeded to perform a more direct test of our hypothesis. We presented the 3 control stimuli (bidirectional stimuli with components at $\pm 30^{\circ}, \pm 40^{\circ}$ or $\pm 50^{\circ}$ ) together with a stimulus comprising 3 directions that contained components moving at $0^{\circ}$ and $\pm 50^{\circ}\left(b_{1}\right.$ in Fig. 3 ). If the visual system indeed finds the best-fitting sum of two Gaussians for this stimulus, subjects should perceive two directions of motion moving in the same directions as in the $\pm 40^{\circ}$ stimulus rather than the $\pm 50^{\circ}$ components actually present. Subjects could reliably discriminate between stimuli with 2 directions at $\pm 30^{\circ}, \pm 40^{\circ}$ and $\pm 50^{\circ}$, but the 2 direction components perceived in the stimulus containing 3 directions were indeed the same as for the $\pm 40^{\circ}$ stimulus with only 2 directions (Fig. 4, left).

We wondered if this inability of the visual system to exclude the influence of movement in the horizontal direction could be overcome if we supplied additional segregation cues. We colored upward- and downward-moving dots red and the horizontally moving ones green. Subjects were asked to judge the direction of the red, upward-moving component. Subjects still judged the perceived upward motion in the stimulus comprising 3 directions to be the same as the one in the $\pm 40^{\circ}$ stimulus (Fig. 4, cen- 
ter). Interestingly, this was not the case when the horizontal motion was put on a different stereoscopic disparity plane (Fig. 4 , right). With this stimulus manipulation, subjects veridically perceived the upward component, and the responses for the stimulus with 3 directions did not statistically differ from those for the $\pm 50^{\circ}$ stimulus.

\section{Discussion}

In summary, we show that the response of direction-selective neurons in MT to transparent motion was well approximated by the scaled sum of their responses to the individual motion components. Most importantly in the context of this study, population activity profiles were singlepeaked for transparent-motion components moving at acute angles. Our ability to perceive multidirectional motion is, therefore, not dependent on multiple peaks in activity profiles across MT neurons; rather, it seems to be based on an analysis of the overall shape of the population response, effectively recovering the Gaussian-shaped profiles of responses to

the individual components underlying the population activity. This is supported by our demonstration of direction metamers, showing an inability of the visual system to recover motion components if identical activity profiles could be created by alternative stimuli with fewer directional components. Our finding that stereoscopic disparity information but not color information helps the visual system to disambiguate these stimuli supports the proposal that perception of direction in our stimuli involves signals from area MT, which contains both disparity-tuned and direction-tuned neurons ${ }^{17-19}$. Such neurons would allow segregation of direction components if they lay at different depths, effectively creating independent population activities for the different planes. Some color information is processed in the context of motion processing ${ }^{20,21}$, but it seems to be insufficient for the segregation of motion components in our displays, presumably because MT neurons are not color-selective enough to support two distinct surface representations based on color information ${ }^{22,23}$.

Our data show that, contrary to previous proposals, the vectors in multidirectional motion do not seem to be encoded individually by separate populations of neurons. Rather, the broad tuning of direction-selective neurons in areas such as MT leads to a population code in which the overall shape of the population activity, and not (at least for acute angles) separable peaks, encodes directions of motion. Using the complete population activity probably also increases signal-to-noise ratios by using all available information ${ }^{4,24}$.

Such an encoding scheme accounts for a number of motion phenomena, especially single-direction ${ }^{9}$ and bidirectional ${ }^{10}$ aftereffects produced by adaptation of subjects with narrow- and wide-angled bidirectional patterns, respectively. Our data also might help account for some results of psychophysical studies using stimuli that combine many directions or speeds $s^{5,25-27}$. Similarly, our data might contribute to an explanation of the perceptual phenomenon of 'motion repulsion' ${ }^{14,28,29}$, a tendency of subjects to overestimate the angle between directions in trans- parent motion patterns, which is strongest for angles of $\sim 30^{\circ}-40^{\circ}$. The population responses to such patterns are somewhat wider than expected from our simple model, but more data and a more formal analysis are required to determine whether this could account for the perceptual errors observed in motion repulsion.

The demonstration of direction metamers also suggests that independent access to the direction components in transparent motion is lost (in the absence of additional cues such as stereoscopic disparity or speed ${ }^{30}$ ), even though the front end of the motion system seems to require local imbalance of motion signals for perception of transparency ${ }^{31,32}$. Stereoscopic disparity, but not color differences, also help to create perceptual transparency in paired random-dot displays ${ }^{33}$.

The inability to use color as a segregation aid does not seem to hold under all circumstances, though. The influence of noise dots on perception by human subjects and monkeys of coherently moving random dot patterns seems to be reduced if they are of a different color ${ }^{20,21}$. A number of differences between previous studies and ours might account for these discrepancies; notably, these include the requirement of subjects to make precise direction judgments in our experiment and the use of long stimulus durations and larger dot sizes in the previous experiments, possibly allowing eccentric tracking of individual points.

It is indeed possible to devise a computational model that can correctly predict the perceived directions ${ }^{34,35}$, including the two directions perceived when viewing our three-direction stimuli, from population activities such as those we report here for MT neurons. Similarly, in some models of motion processing in $\mathrm{MT}^{36,37}$, acute-angled transparent motion results in single-peaked activity profiles.

In summary, using a combination of single-neuron recordings in monkeys and psychophysics in humans, we demonstrated a neural code that supports perception of multiple values without requiring separate neuronal populations to encode the different stimulus values. Given the straightforwardness of this coding scheme, it is probably not restricted to the encoding of 
motion directions. Rather, it might represent a more general approach, suggesting that metamers similar to the ones demonstrated here for the domain of visual motion direction might exist for other sensory domains.

\section{Methods}

Physiology. All animal procedures were approved by the local animal research committee and complied with relevant laws and institutional guidelines. Standard preparation techniques were used for electrophysiological recordings from single neurons in area $\mathrm{MT}^{38}$ of three male macaque monkeys. Cells were characterized as MT neurons based on their receptive field locations, directional tuning and position in the cortex. MT cells were analyzed if they showed directional tuning (responses as a function of stimulus direction were better fit by a Gaussian than by a horizontal line, and responses to the preferred direction were at least fivefold larger than to the null direction).

The monkey initiated each trial by depressing a lever, and had been trained to respond to a change in luminance of a small spot at the top of the fixation cross by releasing the lever. Throughout a trial, the animal's gaze had to stay within $1^{\circ}-1.3^{\circ}$ of visual angle from the fixation cross. If the animal broke fixation or did not respond to the spot's luminance change within the reaction-time window (200-500 ms after the change), the trial was terminated without reward. Only data from correctly completed trials were analyzed.

During a trial, a moving random dot pattern was presented inside the classical receptive field. Its speed and size was matched to the preferred properties of the cell under study. Dots were bright white squares of $0.1^{\circ}$ width presented at a density of 5 dots per square degree on an otherwise dark computer monitor positioned $57 \mathrm{~cm}$ from the animal. For singledirectional patterns, all dots moved coherently in the same direction; for bidirectional stimuli, half of the dots were assigned to each direction. During stimulus presentation, direction(s) continuously changed at a rate of $100^{\circ}$ per $s$ (ref. 39). For our analysis, the value of the spike density profile of the cell's responses (kernel sigma, $30 \mathrm{~ms}$ ) was taken every $50 \mathrm{~ms}$. Control measurements were taken to ensure that our results, especially the absence of two peaks in the population response to acute angled transparent motion, could be replicated using non-changing patterns.

Psychophysics: stimuli. The experiments were performed using an Apple Macintosh computer and a monitor with $74.5 \mathrm{~Hz}$ frame rate. The spatial resolution of the display was 33.3 pixels per degree of visual angle. Our stimuli consisted of random dot patterns (RDPs) presented behind a stationary virtual aperture $6^{\circ}$ in diameter. Each RDP was made up of 200 dots (density, 3 dots per square degree; width, 3.6 arcmin) All dots moved uniformly at $4^{\circ}$ per $\mathrm{s}$ for a stimulus duration of $500 \mathrm{~ms}$, with dots that disappeared behind the aperture reappearing at the opposite side. The subjects viewed the display binocularly in a dimly lit room from a constant distance of $57 \mathrm{~cm}$, maintained by a chin rest. To minimize tracking eye movements, subjects were instructed to maintain fixation on a small cross, $6^{\circ}$ to the left of the stimulus center. Tracking eye movements would change the relative directions of the motion components in the retinal image, destroying the metameric qualities of the stimulus.

The directions in all stimuli were arranged symmetrically around the horizontal direction, moving to either the right or left. (Fig. 3 shows only the rightward moving patterns.) Stimuli with potentially indistinguishable direction components (Fig. 3, $\mathbf{b}_{1}$ and $\mathbf{b}_{2}$ ) were constructed such that a summation of the response profiles created by the two, three or five individual components yielded almost identical single-peaked activity profiles (assuming a bandwidth of the underlying response profiles of $90^{\circ}$ ). Additionally, bidirectional stimuli were used with $\pm 30^{\circ}$ and $\pm 50^{\circ}$ components.

The strength of each individual motion component was scaled multiplicatively by the number of dots moving in that particular direction. The assumption that the neural strength of activation by a particular direction component is a linear function of dot density is a simplification, at least for single-direction patterns ${ }^{3}$. Given that the overall dot density of the stimulus was kept constant and that our simple model predicted approximately identical population activities over a wide range of component dot densities, this simplification probably allowed the model to predict perception successfully nevertheless.
In experiment 1 and 3 , all dots were black; in experiment 2 , the horizontally moving dots were either green or an approximately isoluminant red. In experiment 3 , RDPs were viewed through a mirror stereoscope. The patterns moving obliquely were presented with $0.25^{\circ}$ crossed disparity, whereas the patterns moving horizontally were presented with $0.25^{\circ}$ uncrossed disparity.

Subjects. The same twelve subjects served in all experiments. Nine were paid volunteers naive to the research aim, and three were from within the laboratory. All had normal or corrected-to-normal visual acuity.

Task. Psychophysical testing protocols were approved by the ethics commission of the University of Tübingen Medical School. The different patterns were presented in random order in blocks of 160 trials. Subjects had to report the upward direction component in each pattern. Immediately after the moving dot patterns, a line was presented on the screen until the subject made a decision. In a two-alternative forced choice protocol, subjects were required to indicate whether the perceived steepness of the upward moving component in the RDP was smaller or larger than the slope of the line.

Data analysis. After each trial, in a 50\%-staircase procedure, the line was brought toward the perceived direction of motion. For the value of perceived direction, the results of leftward and rightward motion of all subjects for each of the four stimuli were averaged.

Note: further material and demonstrations of our stimuli can be viewed on the Nature Neuroscience web (http://neurosci.nature.com/web_specials/).

\section{ACKNOWLEDGements}

This work was supported by a grant from the MWF Baden-Württemberg. We are grateful to O. Braddick, N. Qian and R.S. Zemel for comments on previous versions of the manuscript.

RECEIVED 29 DECEMBER 1999; ACCEPTED 25 JANUARY 2000

1. Graziano, M. S. A., Andersen, R. A. \& Snowden, R. J. Tuning of MST neurons to spiral motions. J. Neurosci. 14, 54-67 (1994).

2. Albright, T. D. Direction and orientation selectivity of neurons in visual area MT of the macaque. J. Neurophysiol. 52, 1106-1130 (1984).

3. Snowden, R. J., Treue, S. \& Andersen, R. A. The response of neurons in areas V1 and MT of the alert rhesus monkey to moving random dot patterns. Exp. Brain Res. 88, 389-400 (1992).

4. Britten, K. H. \& Newsome, W. T. Tuning bandwidth for near-threshold stimuli in area MT. J. Neurophysiol. 80, 762-770 (1998).

5. Williams, D. \& Sekuler, R. Coherent global motion percepts from stochastic local motions. Vis. Res. 24, 55-62 (1984).

6. Pasternak, T., Albano, J. E. \& Harvitt, D. M. The role of directionally selective neurons in the perception of global motion. J. Neurosci. 10, 3079-3086 (1990).

7. Jasinschi, R., Rosenfeld, A. \& Sumi, K. Perceptual motion transparency: the role of geometrical information. J. Opt. Soc. Am. A Opt. Image Sci. Vis. 9, 1865-1879 (1992).

8. Mulligan, J. B. Motion transparency is restricted to two planes. Invest. Ophthalmol. Vis. Sci. Suppl. (1992).

9. Verstraten, F. A., Fredericksen, R. E. \& Van de Grind, W. A. Movement aftereffect of bi-vectorial transparent motion. Vis. Res. 34, 349-358 (1994).

10. Grunewald, A. \& Lankheet, M. J. M. Orthogonal motion after-effect illusion predicted by a model of cortical motion processing. Nature 384, 358-360 (1996).

11. Wilson, H. R. \& Kim, J. A model for motion coherence and transparency. Vis. Neurosci. 11, 1205-1220 (1994).

12. van Wezel, R. J. A. et al. Responses of complex cells in area 17 of the cat to bivectorial transparent motion. Vis. Res. 36, 2805-2813 (1996).

13. Recanzone, G. H., Wurtz, R. H. \& Schwarz, U. Responses of MT and MST neurons to one and two moving objects in the receptive field. J. Neurophysiol. 78, 2904-2915 (1997).

14. Mather, G. \& Moulden, B. A simultaneous shift in apparent directions: Further evidence for a 'distribution-shift' model of direction coding. Q. J. Exp. Psychol. 32, 325-333 (1980).

15. Salzman, C. D. \& Newsome, W. T. Neural mechanisms for forming a perceptual decision. Science 264 231-237(1994).

16. Williams, D., Tweten, S. \& Sekuler, R. Using metamers to explore motion perception. Vis. Res. 31, 275-286 (1991). 


\section{articles}

17. Maunsell, J. H. R. \& Van Essen, D. C. Functional properties of neurons in middle temporal visual area of the macaque monkey. II. Binocular interactions and sensitivity to binocular disparity. J. Neurophysiol. 49, 1148-1167 (1983)

18. Bradley, D. C., Qian, N. \& Andersen, R. A. Integration of motion and stereopsis in middle temporal cortical area of macaques. Nature 373, 609-611 (1995).

19. DeAngelis, G. C., Cumming, B. G. \& Newsome, W. T. Cortical area MT and the perception of stereoscopic depth. Nature 394, 677-680 (1998).

20. Croner, L. J. \& Albright, T. D. Image segmentation enhances discrimination of motion in visual noise. Vis. Res. 37, 1415-1427 (1997).

21. Croner, L. J. \& Albright, T. D. Segmentation by color influences responses of motion-sensitive neurons in the cortical middle temporal visual area. J. Neurosci. 19, 3935-3951 (1999).

22. Zeki, S. M. The distribution of wavelength and orientation selective cells in different areas of monkey visual cortex. Proc. R. Soc. Lond. B Biol. Sci. 217, 449-470 (1983).

23. Saito, H., Tanaka, K., Isono, H., Yasuda, M. \& Mikami, A. Directionally selective responses of cells in the middle temporal temporal area (MT) of the macaque monkey to the movement of equiluminous opponent color stimuli. Exp. Brain Res. 75, 1-14 (1989).

24. Deneve, S., Latham, P. E. \& Pouget, A. Reading population codes: A neural implementation of ideal observers. Nat. Neurosci. 2, 740-745 (1999).

25. Watamaniuk, S. N. J. \& Duchon, A. The human visual system averages speed information. Vis. Res. 32, 931-941 (1992).

26. Watamaniuk, S. N. J. Ideal observer for discrimination of the global direction of dynamic random-dot stimuli. J. Opt. Soc. Am. A Opt. Image Sci. Vis. 10, 16-28 (1993).
27. Zohary, E., Scase, M. O. \& Braddick, O. J. Integration across directions in dynamic random dot displays: Vector summation or winner take all? Vis. Res. 36, 2321-2331 (1996).

28. Marshak, W. M. \& Sekuler, R. Mutual repulsion between moving visual targets. Science 205, 1399-1401 (1979).

29. Rauber, H. J. \& Treue, S. Revisiting motion repulsion: Evidence for a general phenomenon. Vis. Res. 39, 3187-3196 (1999).

30. Van Der Smagt, M. J., Verstraten, F. A. J. \& Van De Grind, W. A. A new transparent motion aftereffect. Nat. Neurosci. 2, 595-596 (1999).

31. Qian, N., Andersen, R. A. \& Adelson, E. H. Transparent motion perception as detection of unbalanced motion signals III: Modeling. J. Neurosci. 14, 7381-7392 (1994)

32. Qian, N., Andersen, R. \& Adelson, E. H. Transparent motion perception as detection of unbalanced motion signals I: Psychophysics. J. Neurosci. 14, 7357-7366 (1994).

33. Braddick, O. Local and global representations of velocity: transparency, opponency, and global direction perception. Perception 26, 995-1010 (1997).

34. Zemel, R. S., Dayan, P. \& Pouget, A. Probabilistic interpretation of population codes. Neural Comput. 10, 403-430 (1998).

35. Zemel, R. S. \& Dayan, P. Advances in Neural Information Processing Systems Vol. 11 (MIT Press, Cambridge, Massachusetts, in press).

36. Simoncelli, E. P. \& Heeger, D. J. A model of neural responses in visual area MT. Vis. Res. 38, 743-761 (1998).

37. Wang, R. Y. A network model of motion processing in area MT of primates. J. Comput. Neurosci. 4, 287-308 (1997).

38. Treue, S. \& Maunsell, J. H. R. Effects of attention on the processing of motion in macaque visual cortical areas MT and MST. J. Neurosci. 19, 7603-7616 (1999).

39. Schoppmann, A. \& Hoffmann, K. P. Continuous mapping of direction selectivity in the cat's visual cortex. Neurosci. Lett. 2, 177-181 (1976) 